\title{
Investigating the Role of Organizational Values, Communication Satisfaction and Charismatic Leadership on Job Performance with Emphasis on the Mediating Role of Psychological Components Related to Nurses' Work
}

\author{
Azizeh Pashaei Yousef Kandi ${ }^{*}$ (D), Mohammad Hassani²
}

1. MSc, Department of Educational Sciences, Faculty of Literature and Humanities, Urmia University, Urmia, Iran

2. Professor, Department of Educational Sciences, Faculty of Literature and Humanities, Urmia University, Urmia, Iran

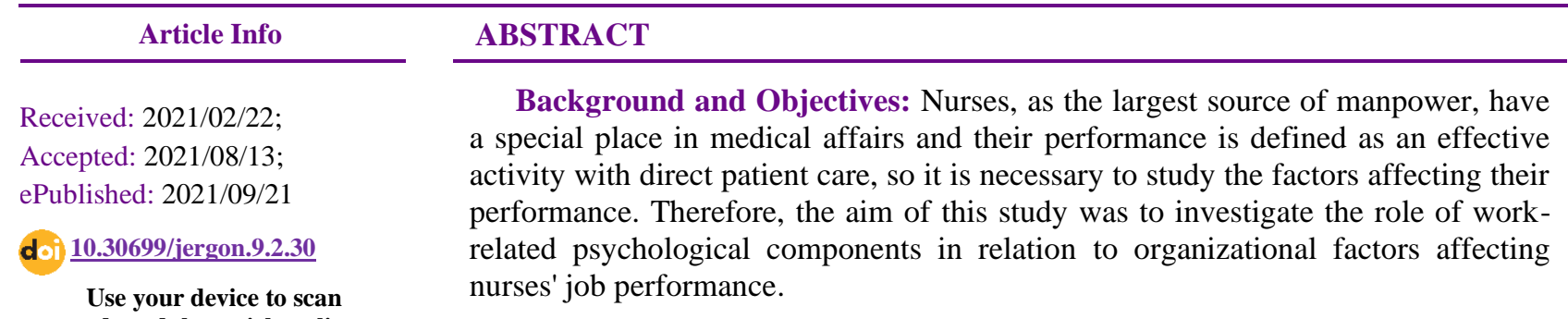
and read the article online

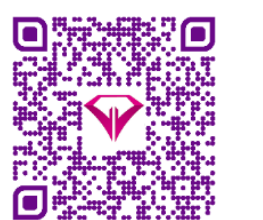

Corresponding Author Azizeh Pashaei Yousef Kandi

MSc, Department of

Educational Administration,

Urmia University, Urmia, Iran

Email:

pashaieazizeh@gmail.com

Methods: This research is a descriptive-correlational study of structural equations according to the data collection method. The statistical population of the present study includes all nurses of Seyed Al-Shohada (AS) Hospital in Urmia, Iran, $(n=265)$. Using Krejcie and Morgan table, 155 people were selected as a statistical sample using relative stratified random sampling method. A questionnaire was used to collect information and after calculating the validity and reliability of the instruments, descriptive and inferential analysis of the data was performed using SPSS 22 and PLS 3 software.

Results: The results of data analysis showed that organizational values, communication satisfaction and charismatic leadership with $\mathrm{t}$-values greater than $1.96(P<0.05)$ mediated by psychological factors have a positive and significant effect on nurses' job performance.

Conclusion: Values, goals, leadership, and communication are factors that go beyond a specific situation and create a sense of satisfaction and performance improvement. Managers increase the ability and capability of nurses by clearly explaining the goals and creating a suitable atmosphere for expressing views, by participating in the affairs of the sense of commitment and ownership, and by holding training courses, and provide the possibility for improving their performance.

Keywords: Psychological components, Organizational values, Communication satisfaction, Charismatic leadership, Job performance, Nursing

Copyright $($ ) 2021, This is an original open-access article distributed under the terms of the Creative Commons Attribution-noncommercial 4.0 International License which permits copy and redistribute of the material just in noncommercial usages with proper citation.

How to Cite This Article:

Pashaei Yousef Kandi A, Hassani M. Investigating the Role of Organizational Values, Communication Satisfaction and Charismatic Leadership on Job Performance with Emphasis on the Mediating Role of Psychological Components Related to Nurses' Work. Iran J Ergon. 2021; 9(2):30-41 


\section{Extended Abstract}

\section{Introduction}

Nurses, as the largest source of manpower, have a special place in medical affairs and their performance is defined as an effective activity with direct patient care, so it is necessary to study the factors affecting their performance. Therefore, the aim of this study was to investigate the role of work-related psychological components in relation to organizational factors affecting nurses' job performance.

\section{Methods}

This research is a descriptive-correlational study of structural equations according to the data collection method. The statistical population of the present study includes all nurses of Seyed AlShohada (AS) Hospital in Urmia, Iran, $(n=265)$. Using Krejcie and Morgan table, 155 people were selected as a statistical sample using relative stratified random sampling method. A questionnaire was used to collect information and after calculating the validity and reliability of the instruments, descriptive and inferential analysis of the data was performed using SPSS 22 and PLS 3 software.

\section{Results}

The results of data analysis showed that organizational values, communication satisfaction and charismatic leadership with t-values greater than $1.96 \quad(P<0.05)$ mediated by psychological factors have a positive and significant effect on nurses' job performance.

\section{Discussion}

The results showed that organizational values have a direct, positive and significant effect on employee empowerment. According to Hosseini results, the variable of organizational values has an effect on human resource empowerment [23]. According to the researches, as long as the goals and values of the organization are clear and acceptable to the individual, he will move with them with all his might, and managers should provide empowerment and training for their subordinates and try to use different methods. Also, based on the results, communication satisfaction has a direct, positive and significant effect on nurses' sense of empowerment. But no research was found consistent with these hypotheses. The leaders of the organization should increase the satisfaction of people's communication with new management methods and financial and economic incentives so that they have a sense of belonging and commitment to the organization and move in the direction of the organization's goals and perform better. According to the results of research, Charismatic leadership has a direct, positive and significant effect on empowerment.

According to the results of the analysis, organizational values and satisfaction with communication and charismatic leadership have a direct, positive and significant effect on commitment. Which is consistent with the results of Malakouti and Yazdani studies as well as the Khaleghkhah and Babaei Menqari [10, 24]. More clear and precise expression of the organizational values and if their acceptance by them, will increase the employees' sense of emotional commitment to the organization, and as a result, they will show more effort and will be more attached to the organization. Managers of organizations should pay attention to relationships and communications within the organization and provide opportunities to improve communication and try to involve employees in decisions and provide areas of satisfaction and increase the commitment and sense of belonging to the organization. The leadership practices of an organization are an important and vital element for an organization.

The results showed that organizational values and satisfaction with communication and charismatic leadership have a direct, positive and significant effect on nurses' sense of psychological ownership, but due to lack of research in this field, no background was found that is consistent with this hypothesis. If the goals and values of the organization are in line with the individual goals of employees, they think of themselves as the owners 
of the organization and do their best to support the positions of the organization and consider the organization as their home and do not give up any effort.

The results of the analysis showed that organizational values and satisfaction with communication and charismatic leadership, empowerment, commitment and ownership have a direct, positive and significant effect on job performance. This research is in line with the research of Farooq and Jaffery, as well as Pingo et al., Mathew et al., and Tadampali, Abdul [25-28]. Huili examined the mediating role of organizational values in performance and creates a new conceptual model for work personality and performance [29]. According to the reports, if the goals and values of the individual and the organization are consistent and organizational values are clear, specific and acceptable to individuals, employees will move in the direction of the goals and will perform well. Khanjankhani et al. suggested that managers, while formulating clear and specific goals, involve employees in formulating organizational goals to achieve the highest level of job performance [7]. As a thought-provoking, inspiring, and moral, the charismatic leader is able to develop emotional bonds with his followers and other leaders that will improve the performance of the individual and the organization. Liu et al. also stated that job satisfaction and participatory organizational atmosphere affect employees' sense of ownership towards the organization [30].

Organizational values, communication satisfaction and Charismatic leadership have a positive and significant effect through mediation of empowerment with job performance. No background was found for empowerment mediation in relation to each of the variables. charismatic leadership has a positive and significant relationship with job performance by mediating organizational commitment. Despite the impact and role of organizational commitment on the relationship between independent variables and performance, no research was found to be consistent with the hypotheses.

According to the results of organizational values, communication satisfaction and Charismatic leadership have a positive and significant relationship with psychological performance mediated by psychological property and charismatic leadership has a positive and significant relationship with $99 \%$ confidence. The results of Farzad and Ranjbar research indicated that transformational leadership has a positive relationship with job performance. The mediating role of psychological ownership in the relationship between transformational leadership and job performance was also acknowledged [31]. As a result, by creating a sense of ownership and motivation to maintain and improve employee ownership and apply transformational leadership, it is possible to increase job performance. But no background was found on the mediating role of ownership in relation to organizational values and performance and satisfaction with communication and performance.

\section{Conclusion}

Values, goals, leadership, and communication are factors that go beyond a specific situation and create a sense of satisfaction and performance improvement. Managers increase the ability and capability of nurses by clearly explaining the goals and creating a suitable atmosphere for expressing views, by participating in the affairs of the sense of commitment and ownership, and by holding training courses, and provide the possibility for improving their performance.

\section{Acknowledgement}

The efforts of the supervisor and all the nurses participating in this study are appreciated.

\section{Conflict of Interest}

The authors declared no conflict of interest. 


\section{مقالة بثزوهشى}

بررسى نقش ارزشهاى سازمانى، رضايت از ارتباطات و رهبرى فرهمند بر عملكرد شغلى با تأكيد بر نقش ميانجى

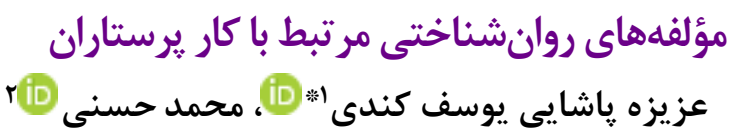

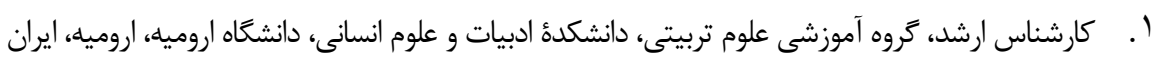

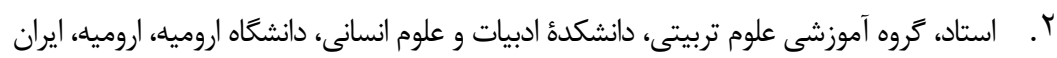

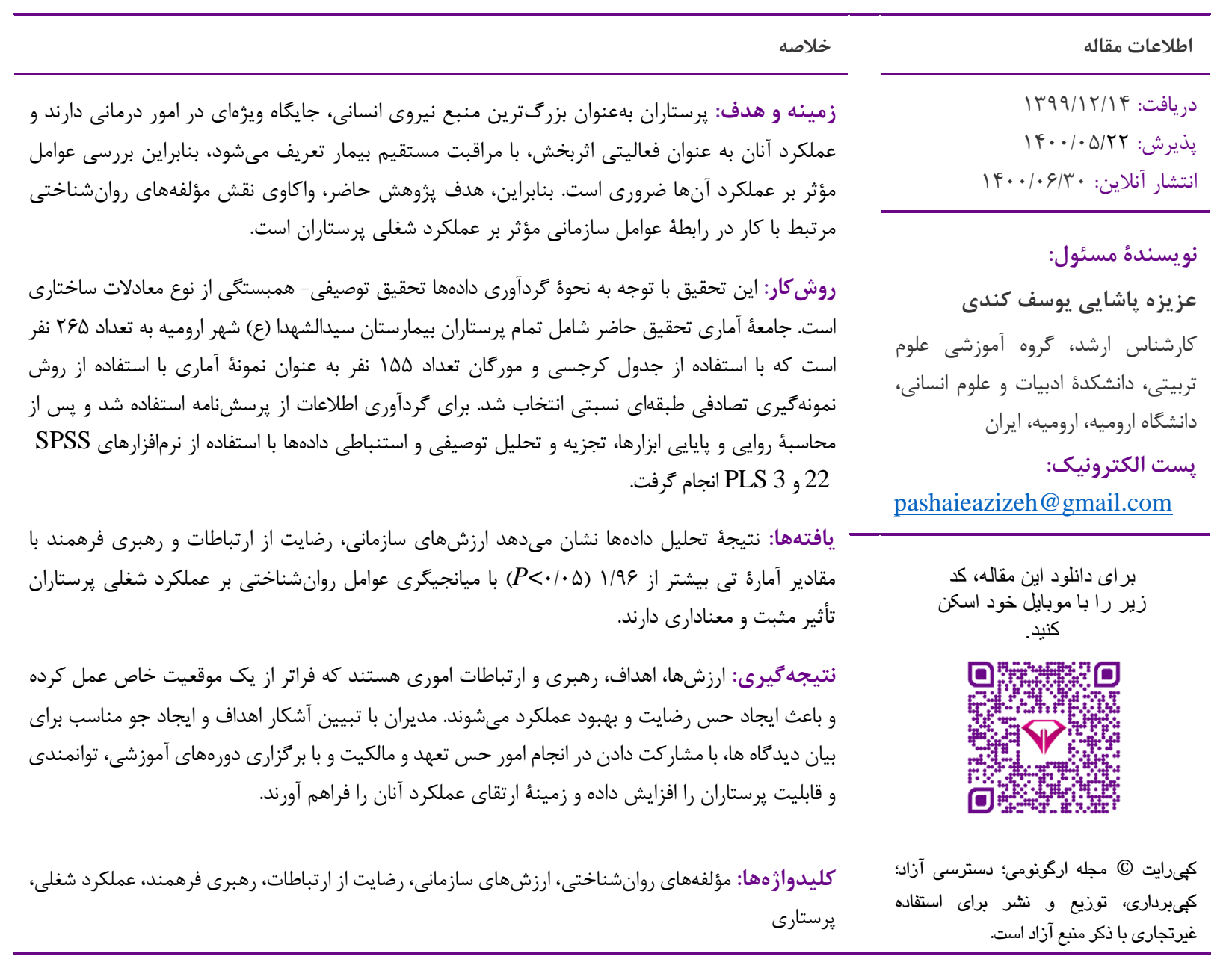

مقدمه

هدف اصلى و غايى حرفه يرستارى تأمين بهبودى و سلامت بشريت

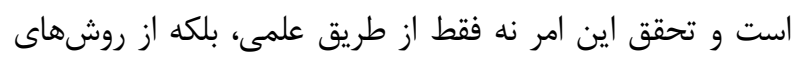

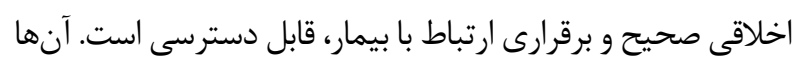

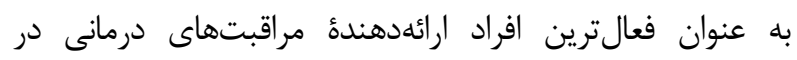

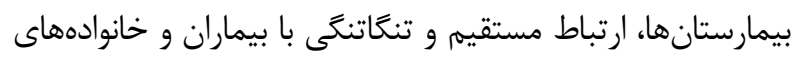
آنان دارند و نقش بسزايى در حفظ، تأمين و ارتقاى سلامتى آنان و ونيان

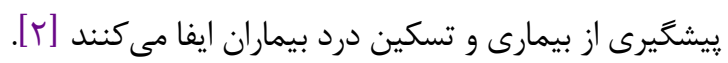

با توجه به اهميت مشاغل خدماتى و ارتباط مستقيم بين

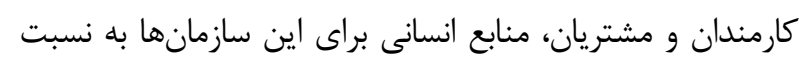

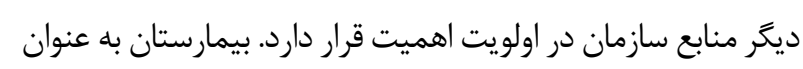

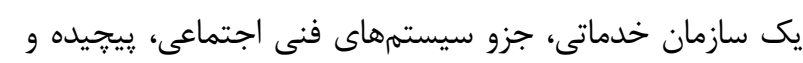

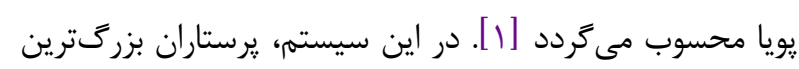

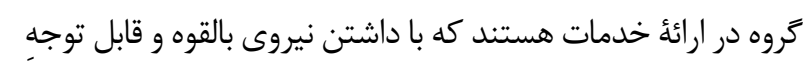

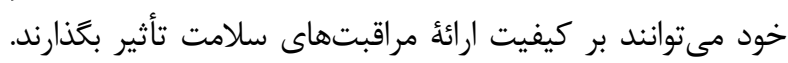


״يش دارند، رهبرىشان را نخواهند يذيرفت. رهبران براى اينكه

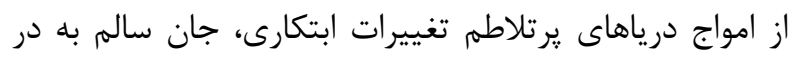

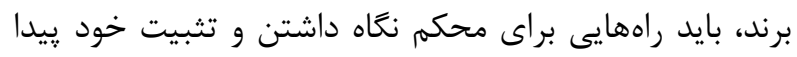

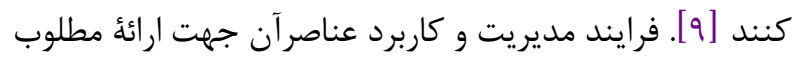

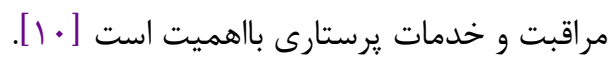

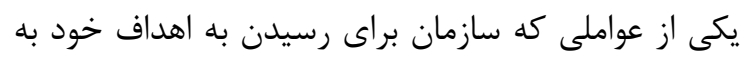

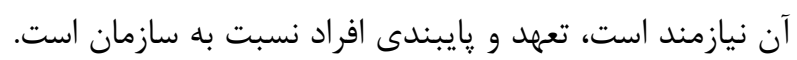

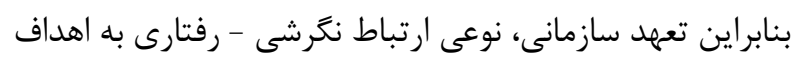

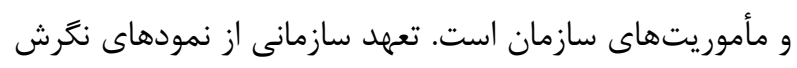

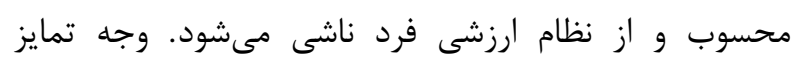

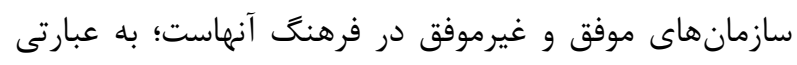

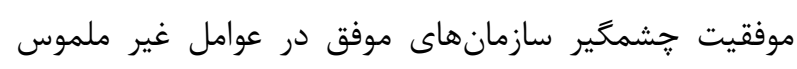

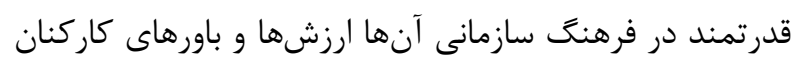

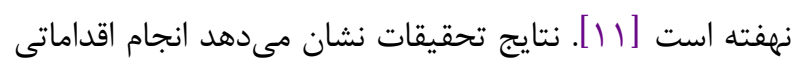
كه منجر به بهبود سطح تعهد عاطفى و هنجارى يرستاران شود

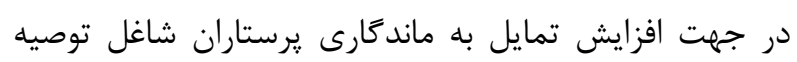

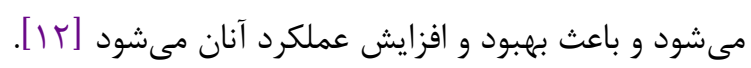

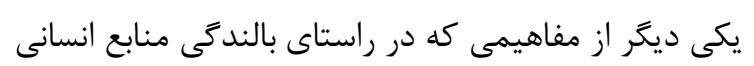

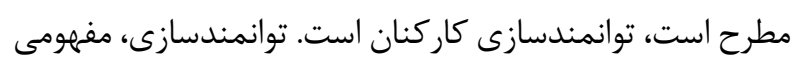
جديد است كه هم اكنون نظر بسيارى از انديشمندان مديريت

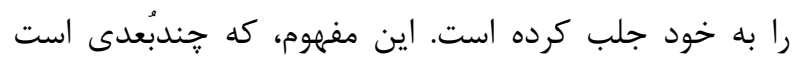

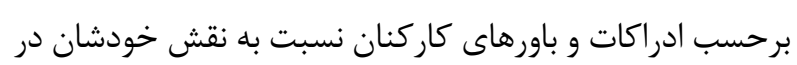

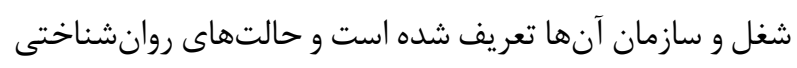
مختلفى را از جمله احساس شايستگى، احساس مؤثر بودن،

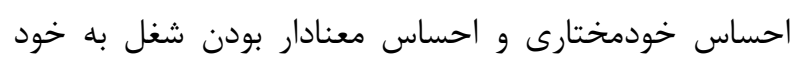

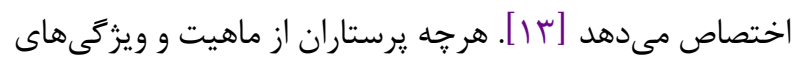
شغلى خود در ابعاد (تنوع مهارت، هويت وظيفه، اهميت وظيفه، آناته استقلال و بازخورد) رضايت بيشتر و مطلوبيت بالاترى داشته إنها

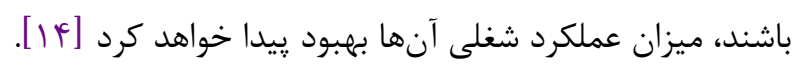

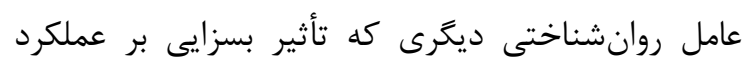

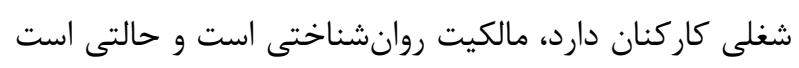

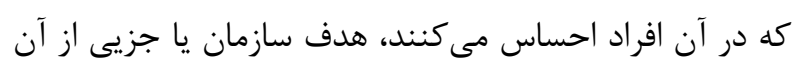

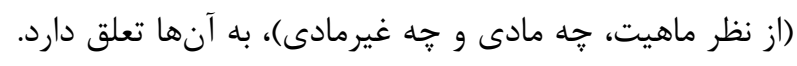

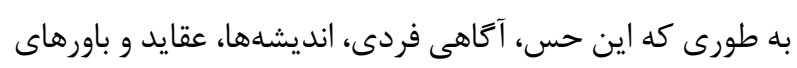

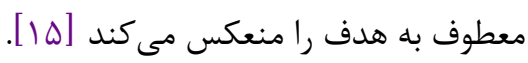

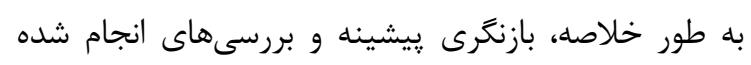
نشان مىدهد تحقيقات داخلى در زمينه ارتقاى عملكرد يرستاران

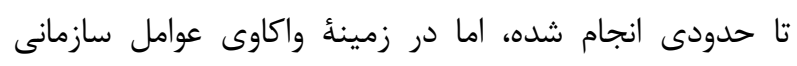

در اين راستا بايد عوامل مؤثر بر عملكرد شغلى ثرستاران

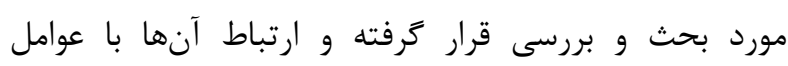

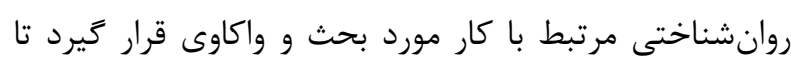

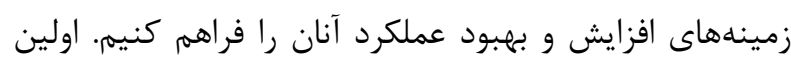

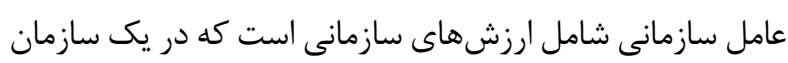

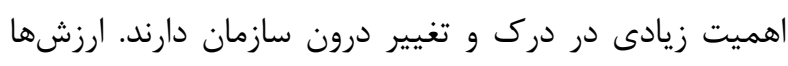

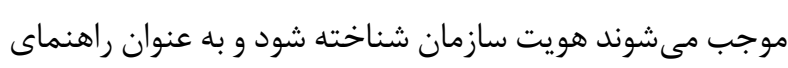

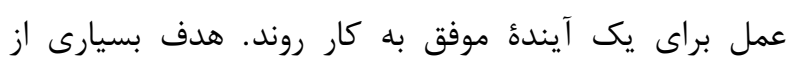

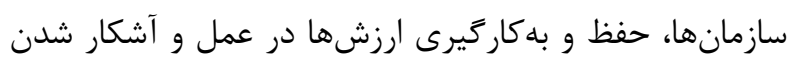

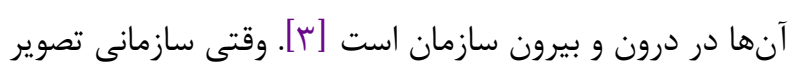

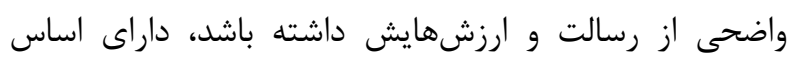

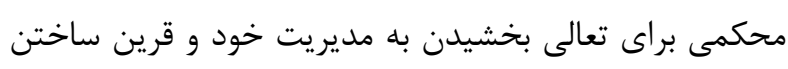

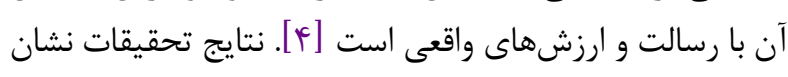

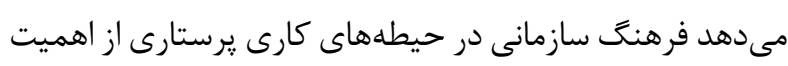

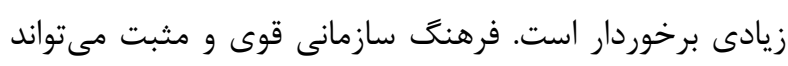

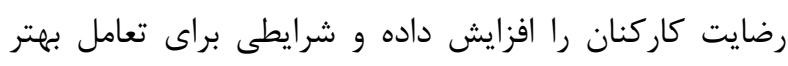

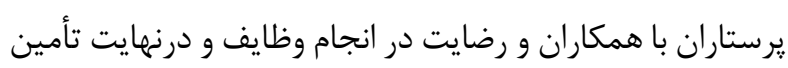

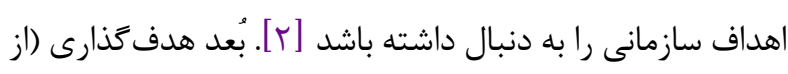

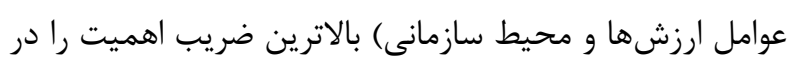

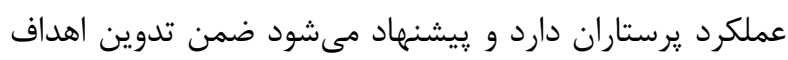

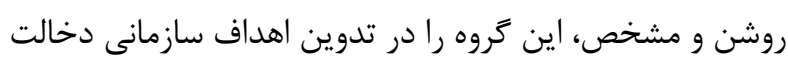

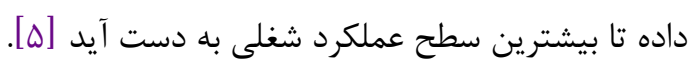

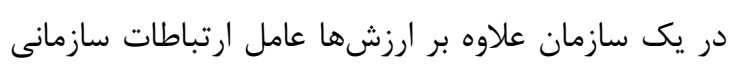

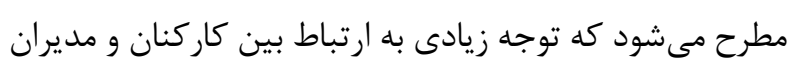

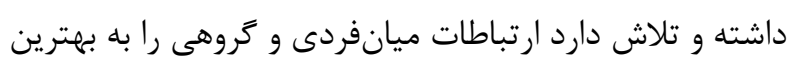

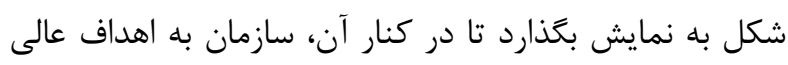

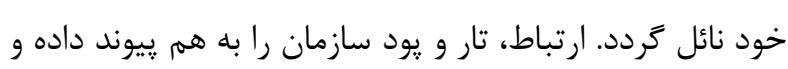

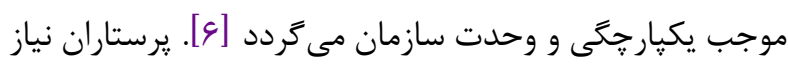

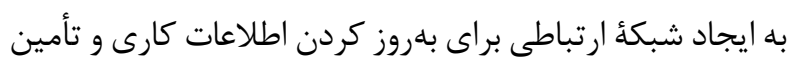

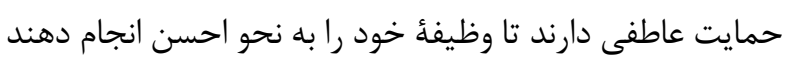

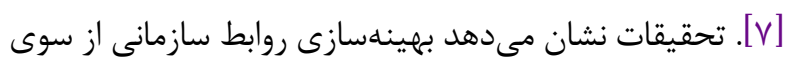

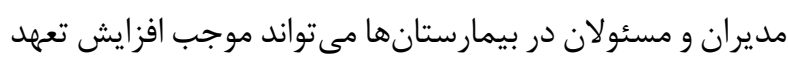

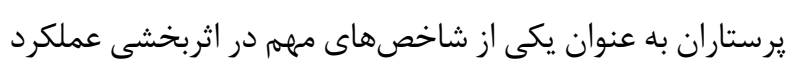

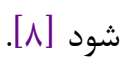
رهبرى در سازمان، تجلى عالىترين شكل مناسبات

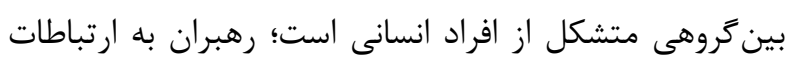

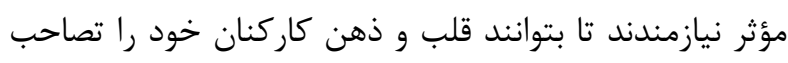

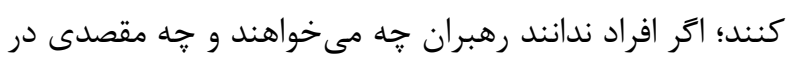


استفاده شده است كه شامل Qf Quinn

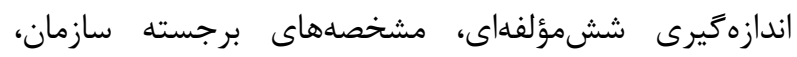

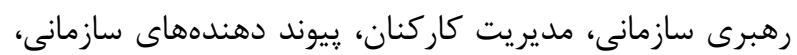

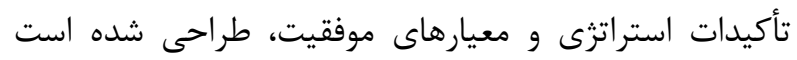

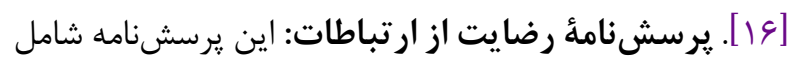

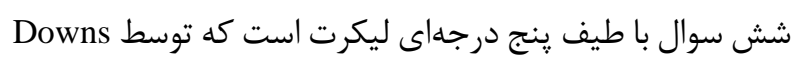

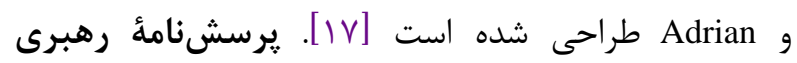

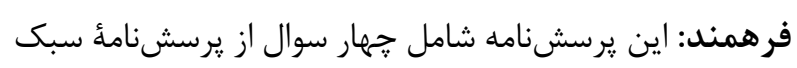
رهبرى Bass و Bvolio

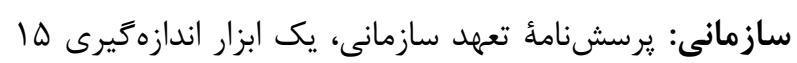

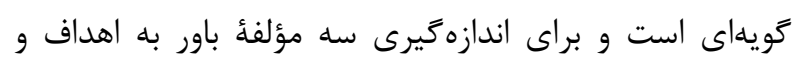
ارزشهاى سازمان، تمايل به تلاش بيشتر در راه سازمان و و تمايل

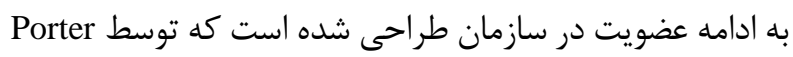

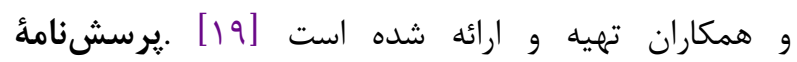

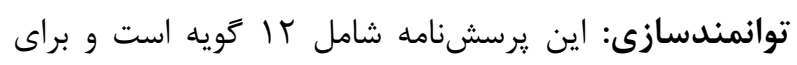
اندازهخيرى جهار مؤلفه معنا، صلاحيت، خودمختارى و تأثير،

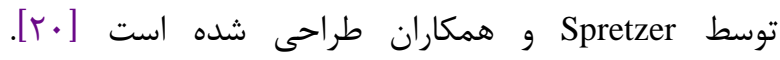

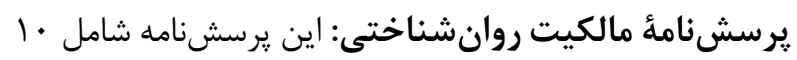

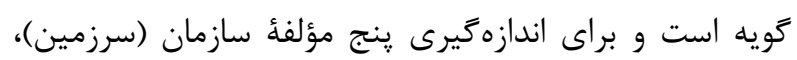

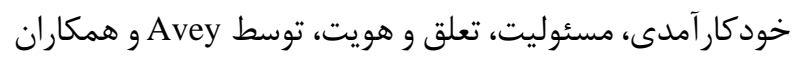

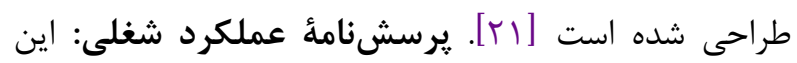

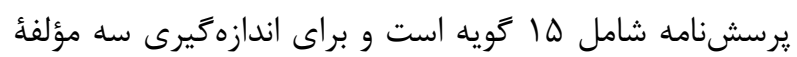

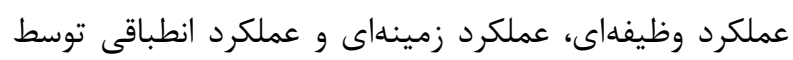
Paterson

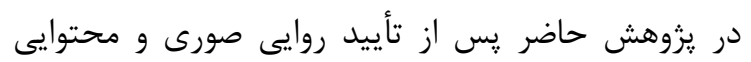

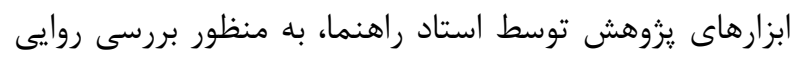

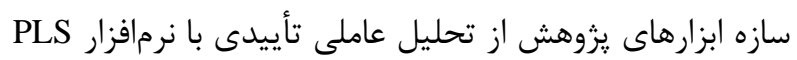

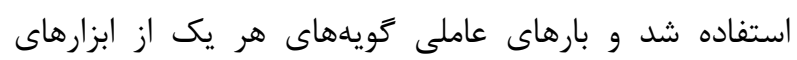

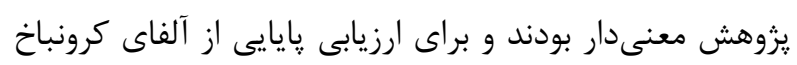

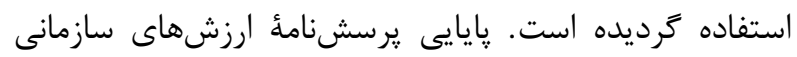

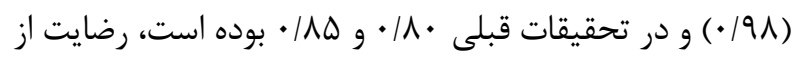

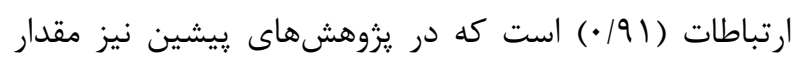

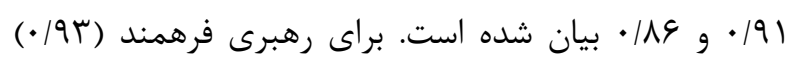

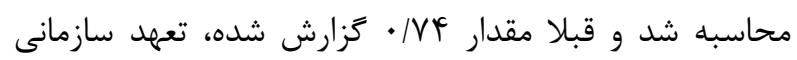

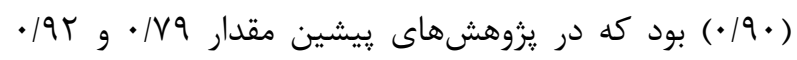

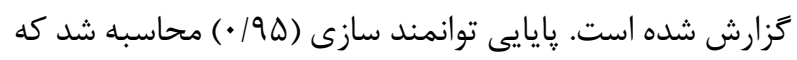

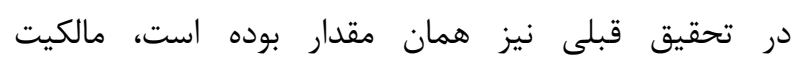

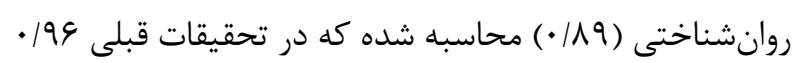

تأثيركذار بر عوامل روانشناختى كه باعث تأثير مضاعفى بر

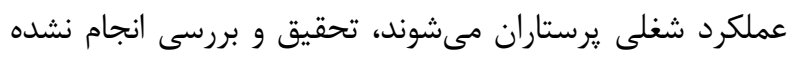

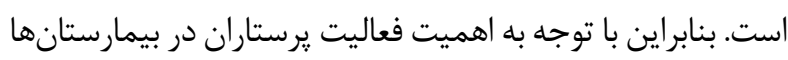

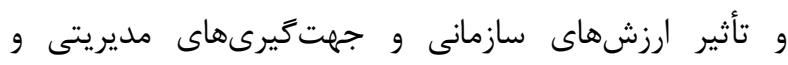

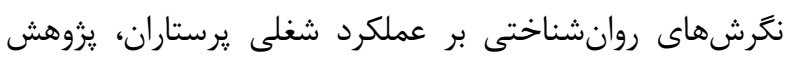

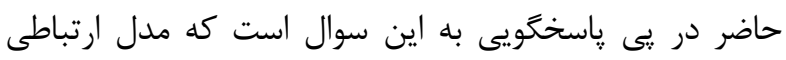

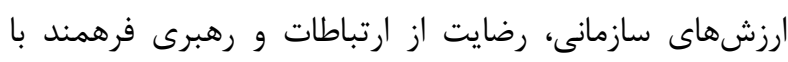

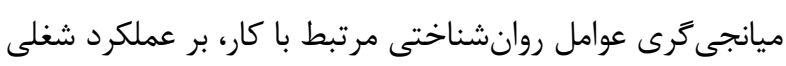

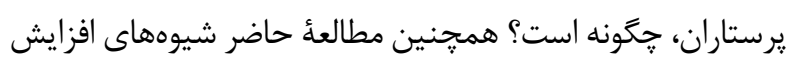

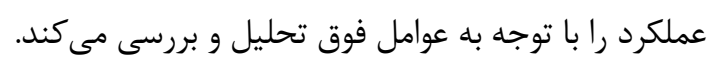

روش كار

يزوهش حاضر از نظر هدف، كاربردى و از نظر نحوه گردآورى

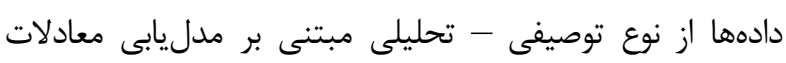

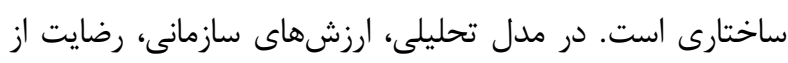

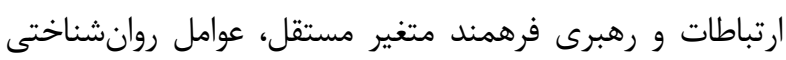

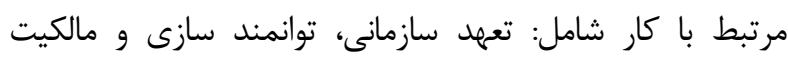

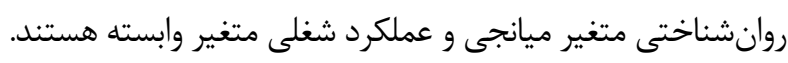

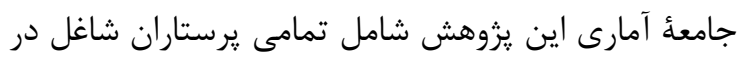

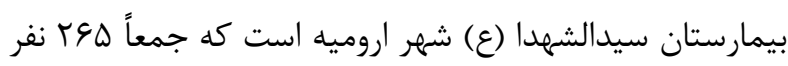

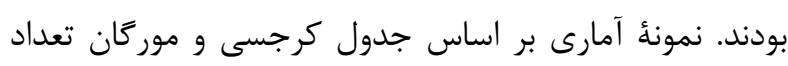

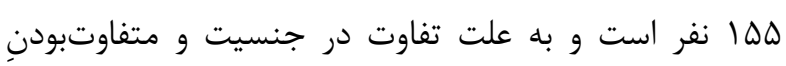

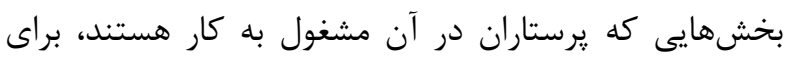

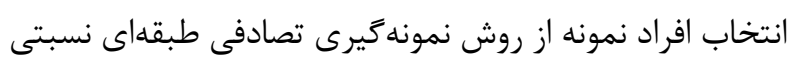

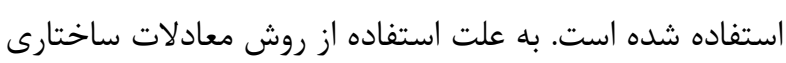

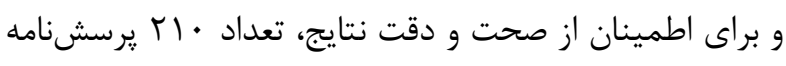

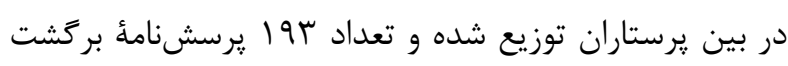

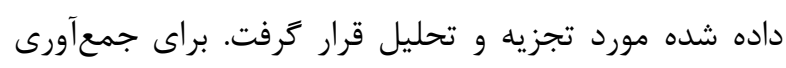

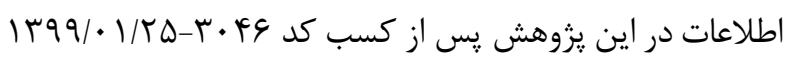

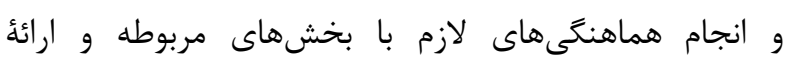

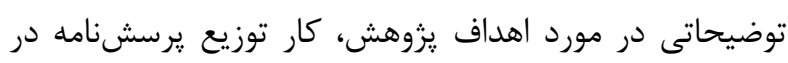

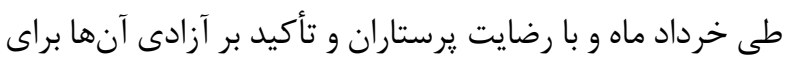

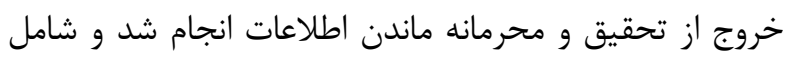

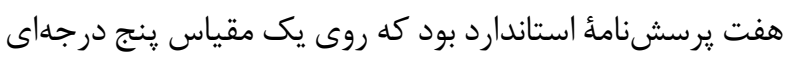

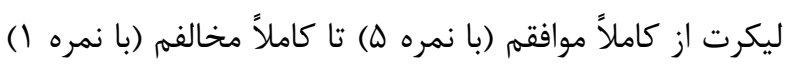
رتبهبندى شده بودند كه شامل موارد زير است:

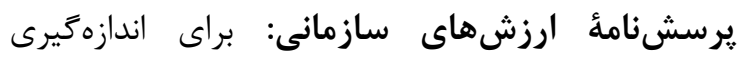

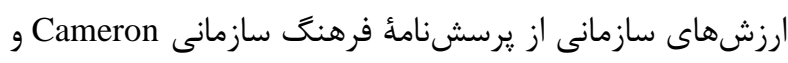




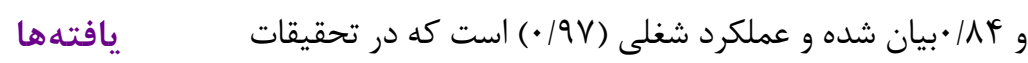

نتايج بررسى اطلاعات جمعيت شناختى آزمودنىهاى

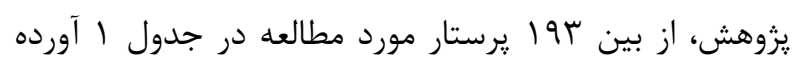

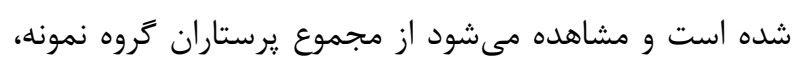

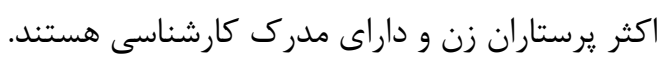

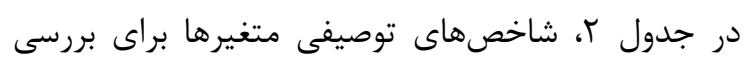

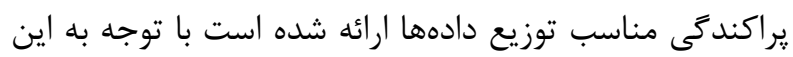

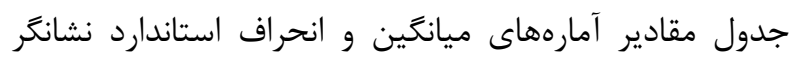
يراكندگى مناسب دادها هستند.

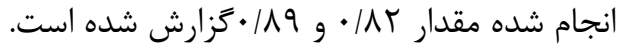

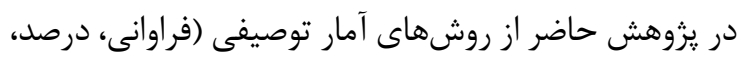

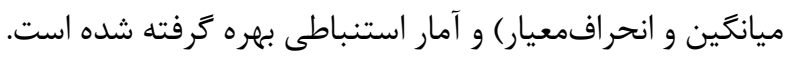

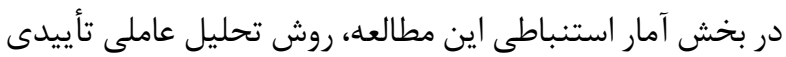
و مدل يابى معادلات ساختارى براى بررسى روابط بين متغيرها

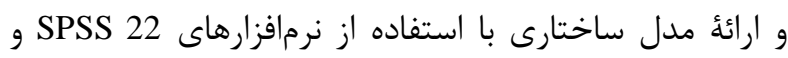
PLS 3 جدول ا. ويثرَى هاى دموكر افيك آزمودنى هاى يُوهش

\begin{tabular}{|c|c|c|}
\hline 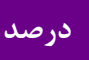 & 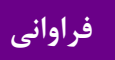 & شاخص آمارى جنسيت \\
\hline$r$. & rı & 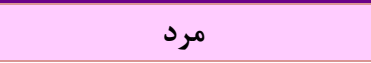 \\
\hline$\wedge$. & $1 \Delta \Delta$ & 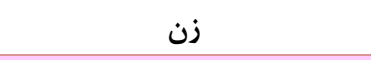 \\
\hline 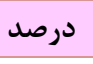 & فراوانى & شاخص آمارى مدرى تحصيلى \\
\hline$\cdot / \Delta$ & 1 & ديبلم \\
\hline$\Delta$ & 9 & كاردانى \\
\hline 91 & IVQ & 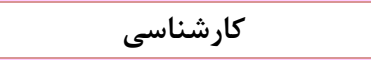 \\
\hline r & $\wedge$ & 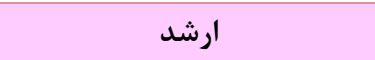 \\
\hline درصد & فراوانى & شاخص آمارى سابقه خدمت \\
\hline 14 & tr & ه-1 اسال \\
\hline r) & 41 & 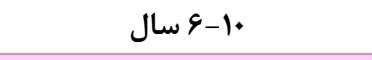 \\
\hline tr & fr & ها-11 سال \\
\hline rI & r. & 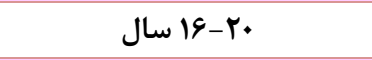 \\
\hline tr & kt & ا ll سال به بالا \\
\hline
\end{tabular}

\begin{tabular}{|c|c|c|c|c|c|c|c|}
\hline 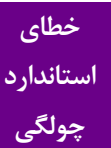 & جولكى & حداكثر & حداقل & انحرافمعيار & ميانكَين & تعداد نمونه & متغير \\
\hline$\cdot / / V \Delta$ & $-\cdot / V^{C}$ & $\Delta$ & $1 / 9 V$ & $\cdot / \mathrm{Vr}$ & $r / 9 \Delta$ & 194 & ارزشهاى سازمانى \\
\hline$\cdot / / v \Delta$ & $-1 / \Delta T$ & F/Ar & $1 / 11$ & $.19 \mathrm{~V}$ & $r / \Lambda r$ & 194 & رضايت از ارتباطات \\
\hline$\cdot / / v \Delta$ & $-1 / \Delta V$ & r/qr & $1 / \Delta \Delta$ &.$|9|$ & $r / v \wedge$ & 194 & رهبرى فرهمند \\
\hline$\cdot / / v a$ & $-1 / 4 v$ & $\Delta$ & 1 & $.19 \mathrm{~V}$ & $r / V r$ & 194 & توانمندسازى \\
\hline$\cdot / / V \Delta$ & $-1 / r \mid$ & $\Delta$ & $1 / 4$ & $\cdot / v$ & $r / v q$ & 194 & تعهد \\
\hline ./IVa & $-1 / r \mid$ & F/Ar & $1 / 9 V$ & $\cdot \mid \Delta \Lambda$ & $r / 99$ & 194 & مالكيت \\
\hline$\cdot / / v \Delta$ & $-1 / 49$ & $F / \Lambda 9$ & $1 / 01$ & .194 & r/AV & 194 & عملكرد شغلى \\
\hline
\end{tabular}




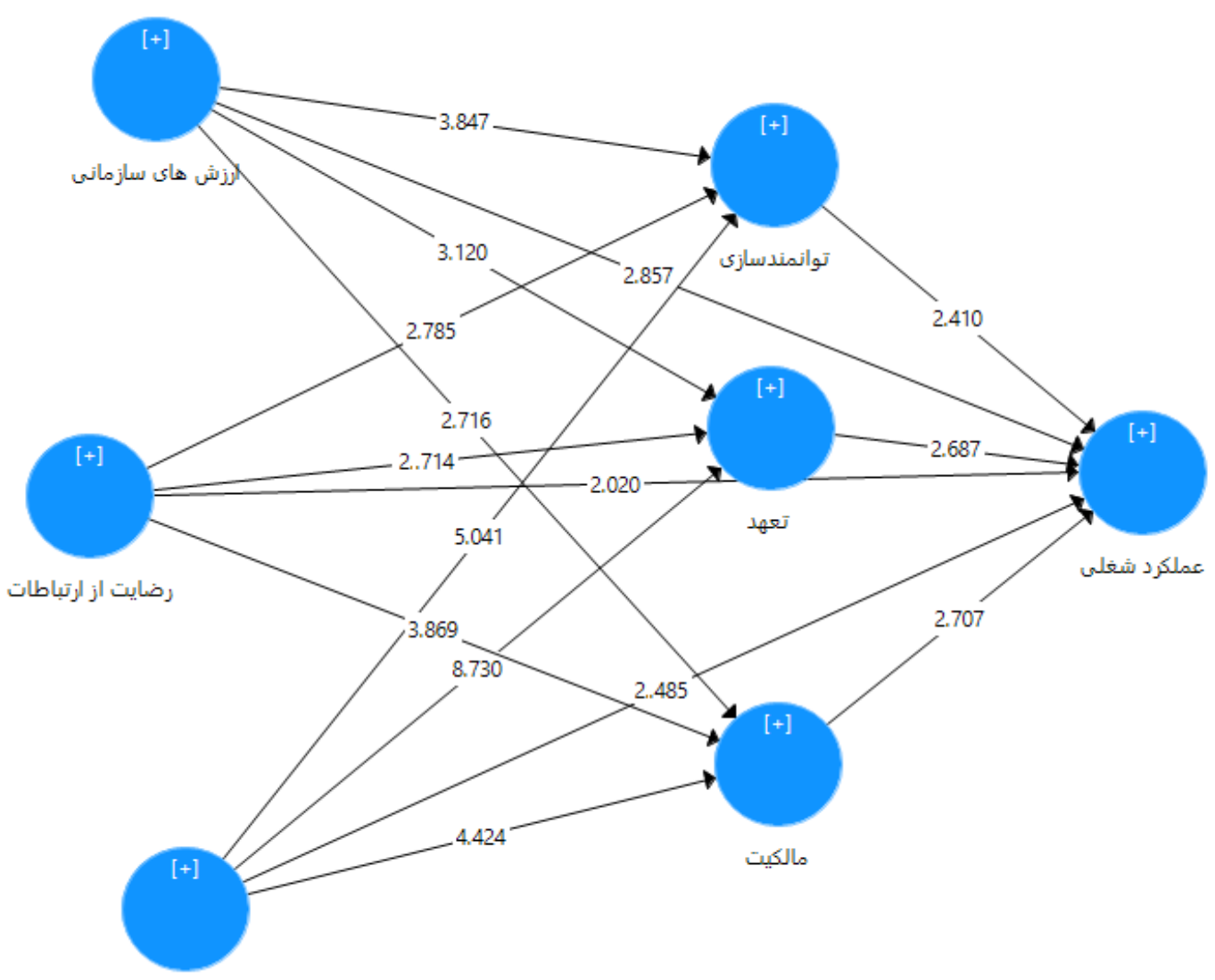

رهبرى كاريزماتيك

t شكل ا. مدل يزوهش در حالت ضرايب

به منظور بررسى فرضيؤ اصلى تحقيق متناسب با جهار جوب نرمافزار PLS استفاده ترديد و نيكويى برازش اين مدل مورد

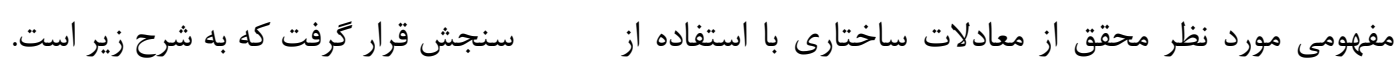

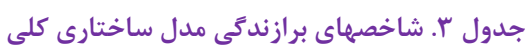

\begin{tabular}{|c|c|c|c|}
\hline شاخص برازش & مقدار مشاهده شده & مقدار مطلوب & نتيجه برازش \\
\hline SRMR & $\cdot / \cdot v$ & كمتر از 1 •|• & مناسب \\
\hline NIF & .194 & بيشتراز • 9/ • & مناسب \\
\hline RMS &.$/ 4 t$ & نزديك به صفر & مناسب \\
\hline d-G & $\cdot 1 \cdot f$ & $<\% 90$ & مناسب \\
\hline d- ULS & $\cdot / \cdot r$ & $<\% 9 \Delta$ & مناسب \\
\hline
\end{tabular}

همجنين مقدار ضرايب تى هريك از مسيرها از متغيرهاى

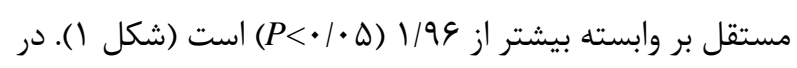
نتيجه، فرضيه كلى تحقيق تأييد مىشود و مى موان كَت

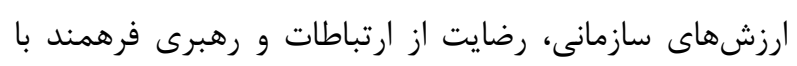
ميانجى زرى عوامل روانشناختى بر عملكرد شغلى تأثير دارند. راند
شاخصهاى برازش مدل عبارتند از SRMR (ريشه

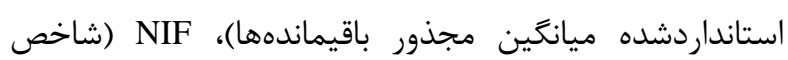
نُرمشدهُ برازش)، RMS (ريشهُ ميانخين مجذورات). با توجه به ميه جدول بالا مقايسه مقادير مطلوب و مقادير مشاهده شده نشان

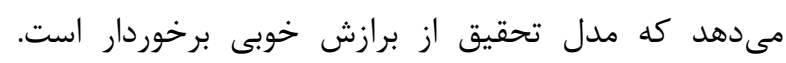


جدول F. جدول بر آورد ضر ايب اثرات مستقيم

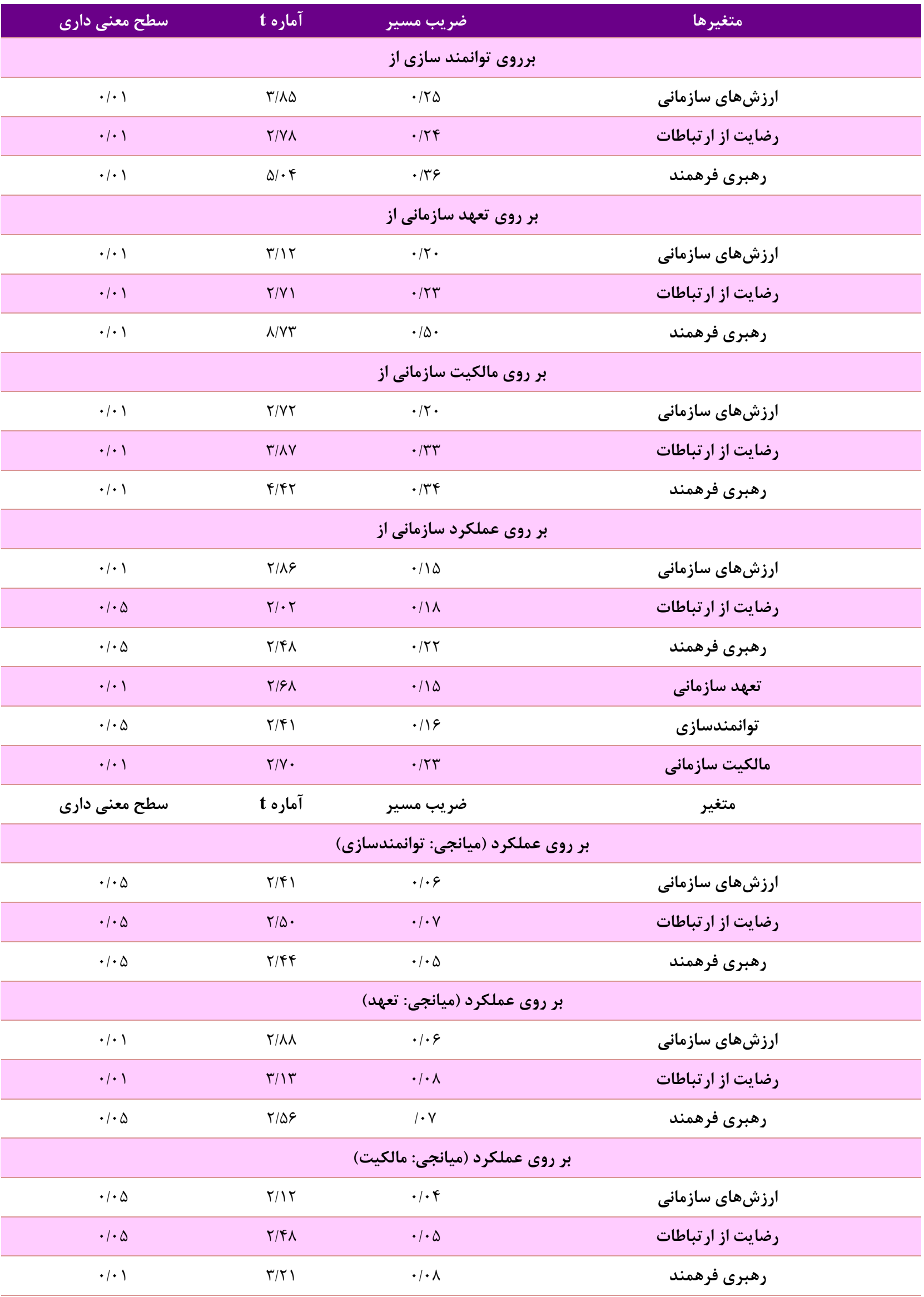


معنادارى دارد، اما بهعلت كمبود تحقيقات در اين زمينه يِيشينداى كه

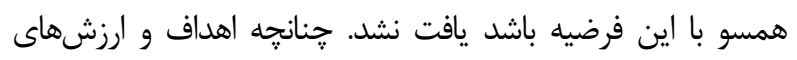

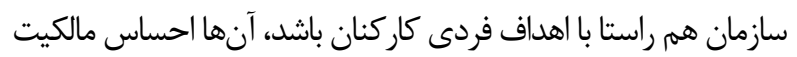

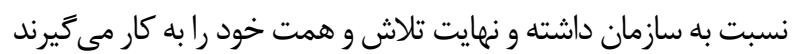

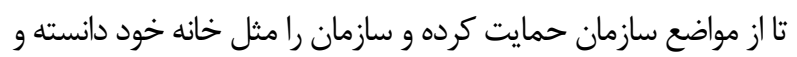
از هيج كوششى فرو تذارى نمى كنند.

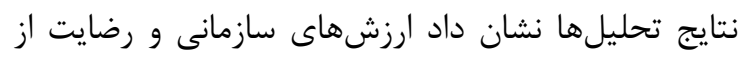

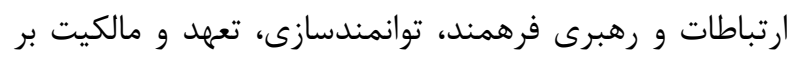

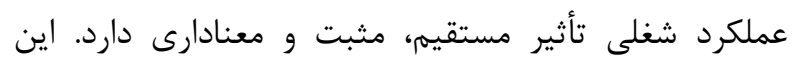

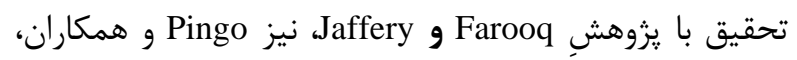

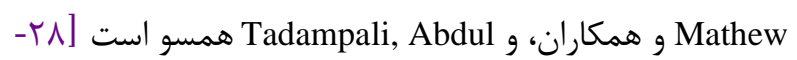
Huili [ro به بررسى نقش واسطهاى ارزشهاى سازمانى بر و

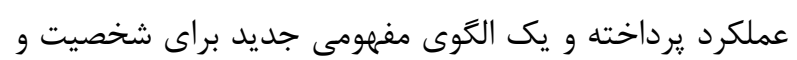

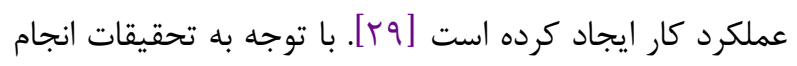

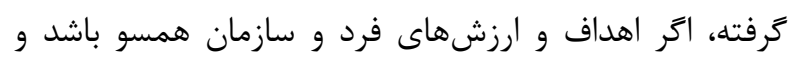

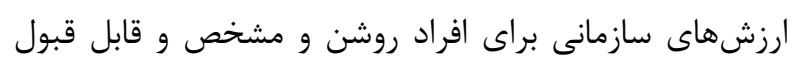

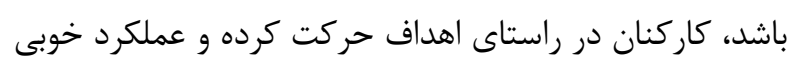

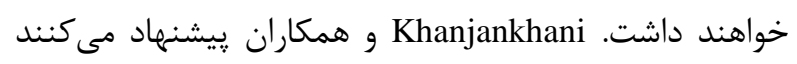

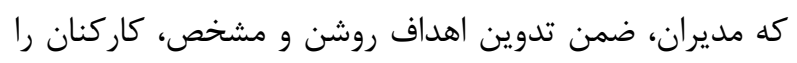

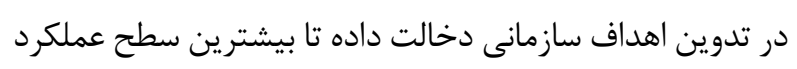

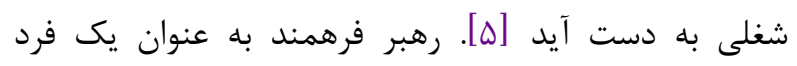

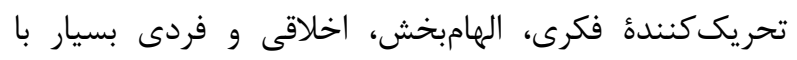

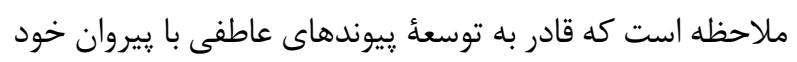

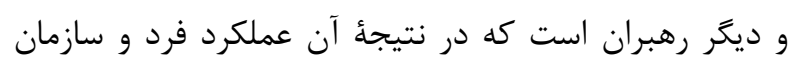

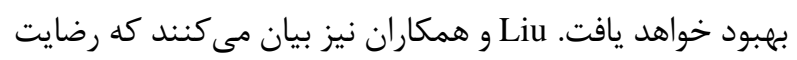
شغلى و جو سازمانى مشاركتى بر احساس مالكيت كاركنان

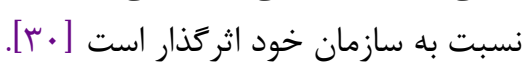

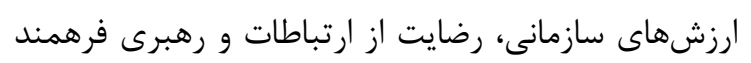

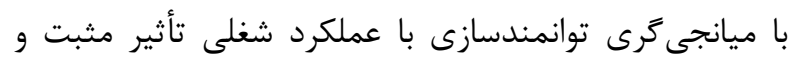

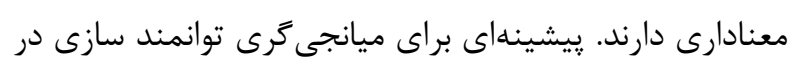

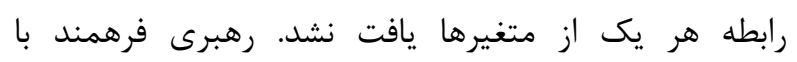
ميانجى

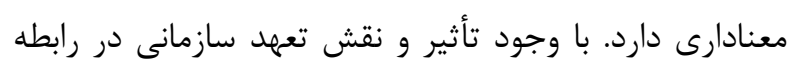

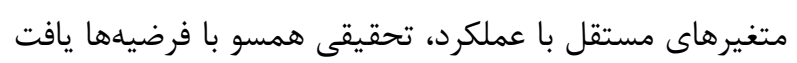

با توجه به نتايج ارزشهاى سازمانى، رضايت از ارتباطات و

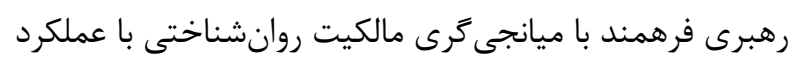

نتايج نشان داد ارزشهاى سازمانى بر توانمندسازى كاركنان

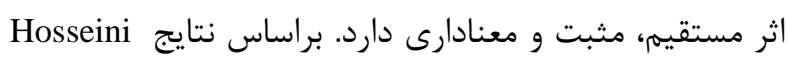

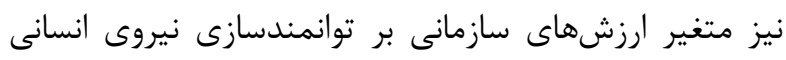

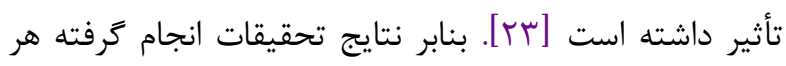

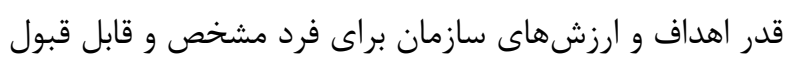

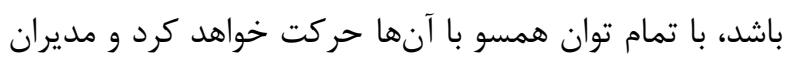

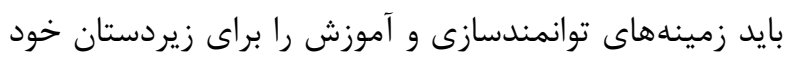

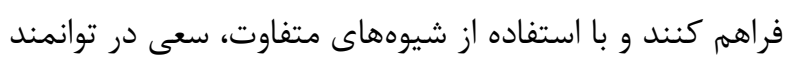

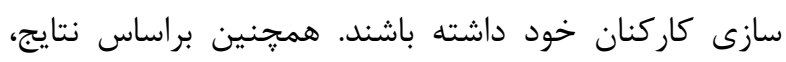

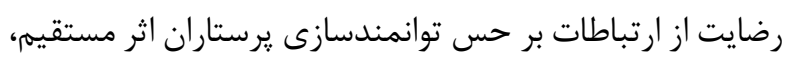

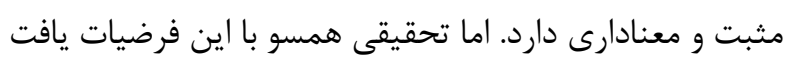

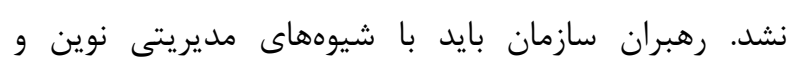

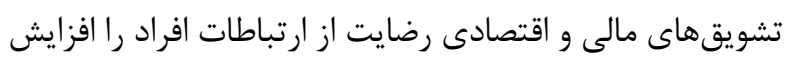

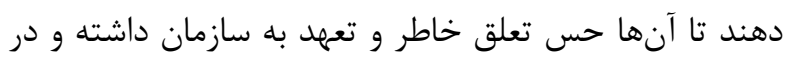

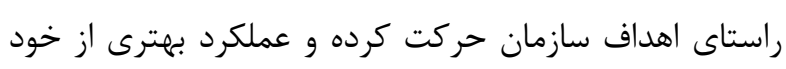

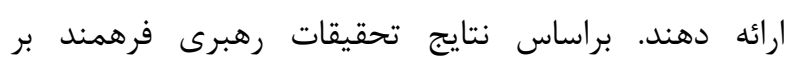
توانمندسازى تأثير مستقيم، مثبت و معنادارى دارد.

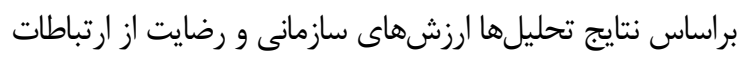

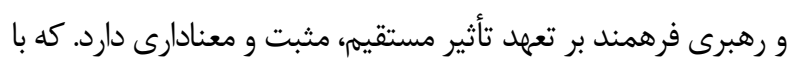

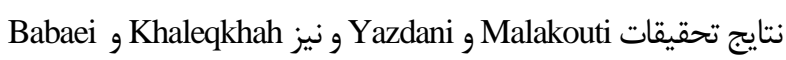
Menqari

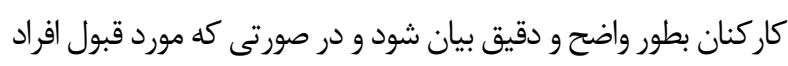
باشد، باعث مىشود كه حس تعهد عاطفى كاركنان نسبت بـ به سازمان

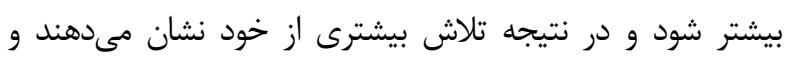

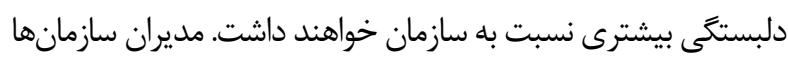

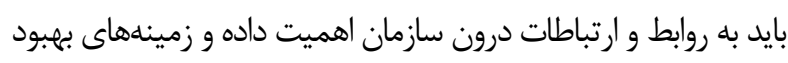

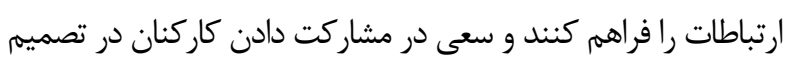

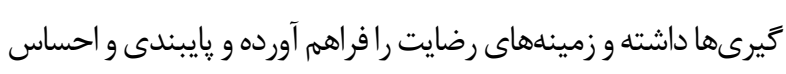

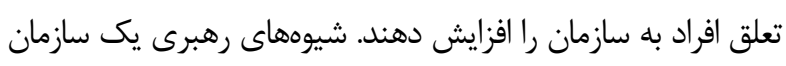

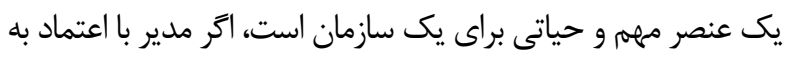

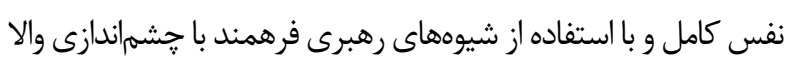

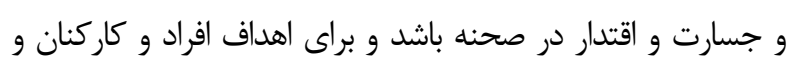
سازمان تلاش كند، واضح و مسله است كه زيردستان او نيز به به همان ونان

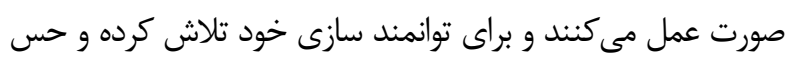
وابستگى و دلبستخى نسبت به سازمان خواهند داشت.

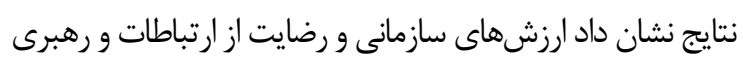

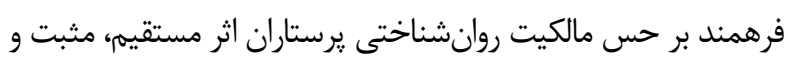


را فراهم آورده و به يرستاران در جارجوب وظايفشان اختيارات

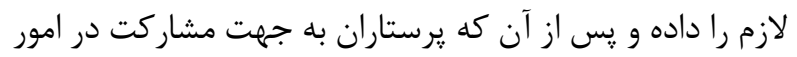

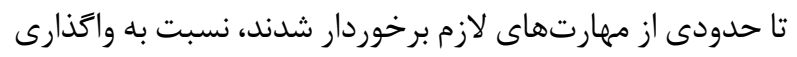

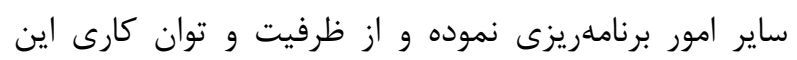

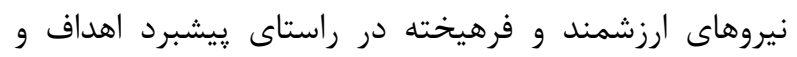
كارايى بهرهمند شوند. اين يزوهش نيز مثل يزوهشهاى ديخر محدوديتهايى داشته است از جمله اينكه در زمان بيمارى كرونا انجام شده و ونديز

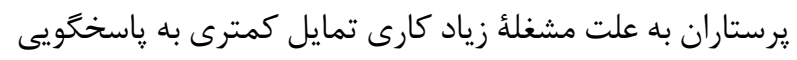

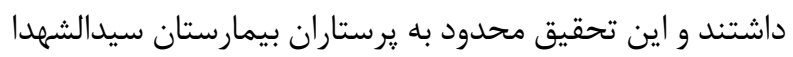
(ع) شهر اروميد است و تعميم نتايج بايد با احتياط انجام شود.

$$
\text { تقدير و تشكر }
$$

$$
\begin{aligned}
& \text { از زحمات استاد راهنما و تمامى يرستاران شركت كننده در } \\
& \text { اين تحقيق، تشكر و قدردانى مىشود. } \\
& \text { سمهم نويسند }
\end{aligned}
$$

سهرم نويسند

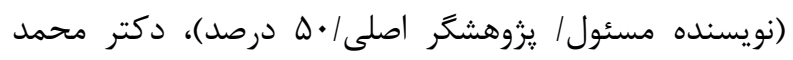

$$
\text { حسنى (نويسنده دوم/ • له درصد). }
$$

$$
\text { تعارض منافع }
$$

بين نويسند كان هيجگُونه تعارض منافعى وجود ندارد.

منابع مالى منابع مالى اين مطالعه توسط نويسندكان تأمين شده است.

\section{References}

1. Zakerian SA, Mosaferchi S, Sepidarkish M, Nasiri Z, Momenyan S, Mortezapour soufiani A. et al. The Role of Individual Effective Factors on Nurses' Job Performance. A Case Study: Selected Hospitals in Tehran Occup Med Quarterly J. 2018;10 (1):54-61.

2. Darvar Alidadi H. The effect of organizational culture and communication skills on job performance (Case study: nurses of Zahedan Social Security Hospital). Master Thesis in Public Management, Human Resource, Zahedan: Sistan and Baluchestan University. 2017.

3. Grossgrove J, Scheer SD, Conklin NL, Jones JM, Safrit, RD. Organizational values perceived as evident among Ohio State University extention personnel. J Ext. 2005;43(5).
شغلى رابطه مثبت و معنادارى دارند و رهبرى فرهمند به

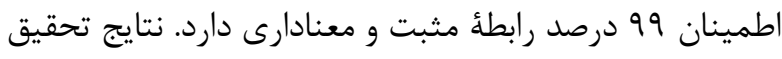
Farzad عملكرد شغلى رابطهاى مثبت دارد. همجنين نقش ميانجى گرى مالكيت روانشناختى در ارتباط بين رهبرى تحول زرا با عملكرد

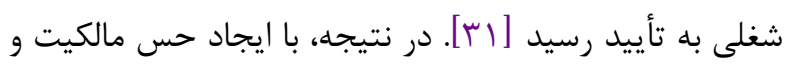
انخيزه حفظ و بهبود مالكيت كاركنان و اعمال رهبرى تحول مى توان زمينهُ افزايش عملكرد شغلى را فراهم كرد. ولى در مر مورد نقش ميانجى مالكيت در رابطه ارزش هاى سازمانى و عملكرد و رضايت از ارتباطات و عملكرد ييشينهاى يافت نشد.

$$
\text { نتيجه كيرى }
$$

ارزشها، مفاهيم، عقايد، اهداف و رهبرى و ارتباطات امورى

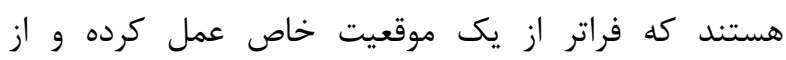
ويزگى هاى بارزى هستند كه عملكرد يرستاران را تحت تأثير قرار داده و باعث افزايش كارايى و اثربخشى آنان ميى هـوند. از

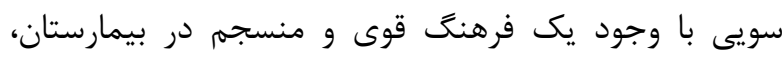
يرستاران ضمن كسب آكاهى بيشتر ييرامون استراتثىهاى بيمارستان، نسبت به ارزشها و هنجارها، احساس مسئوليت و ورن

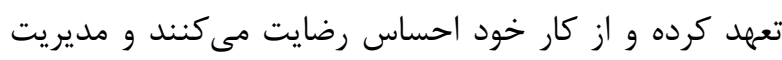
قوى موجب بهبود روحيه، انگَيزش و بهبود عملكرد آنان

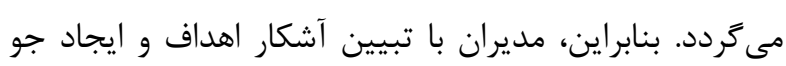

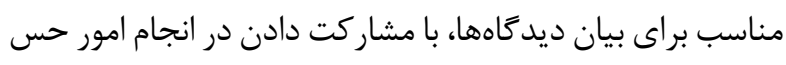

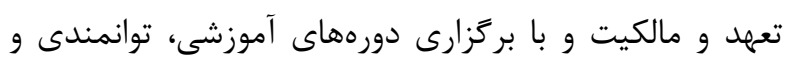
قابليت يرستاران خود را افزايش داده و زمينهُ ارتقا عملكرد آنان

4. Blanchard K, O'Connor, M. Management based on values, translated by Kamran Parvaneh, Tehran: Abangah Publications. 2003.

5. Khanjankhani Kh, Askari R, Askari Shahi M, Shafi'i M. Ranking the dimensions of the internal organizational environment and the five personality traits in the professional performance of nurses in a teaching hospital. J Urmia Nurs Midwifery. 2018;16(5):324-34.

6. Khoshnevis N. Effectiveness of organizational communication. Scientific-Specialized Monthly of Iranian Public Relations Association. 2015; 96:24-30.

7. Babamiri M, Heidari Moghadam R, Mohammadi B, Mohammadi Y. An Investigation on the Relationship between Proactive Personality with Networking, Mediated by Job Satisfaction. Iran J Ergon. 2017;5(3):507. [DOI:10.30699/jergon.5.3.50] 
8. Ghiyasvandian S, Sedighiyani A, Kazemnejad A, Iranshahi I. Relationship between organizational communication satisfaction and organizational commitment in nurses . Med History History Med. 2017; $9(6): 20-33$

9. Khaleqkhah A, Babaei Manqari MM. Relationship between organizational commitment, organizational culture and leadership style. Dev Quarterly. 2015; 36:116.

10. Akbarbegloo M, Bayrami R. Barriers to improving the quality of nursing services from the perspective of employed nurses. Sci J Hamadan School Nurs Midwifery. 2010;18(2):43-49.

11. Ziaei MS, Nargesian A, Adibaghi Isfahani S. The role of spiritual leadership in empowering the staff of the University of Tehran. J Public Manag. 2011;1(1):67-86.

12. Zahedi SM, Qajariyeh F. Relationship between organizational commitment and the tendency to retain nurses working in the Army of the Islamic Republic of Iran. J Nurs Care Res Center of Tehran University of Med Sci (Iranian Journal of Nursing). 2011;24(73):19-26.

13. Razdar MR, Shokhmgar F, Keshtgar M. Investigating the relationship between transformational leadership and psychological empowerment of Ghaen Azad University staff. First National Conference on Manag Econ. 2016.

14. Rahimi H. The Effect of Job Nature on Job Adjustment of Nurses in Hospital Affiliated to Kashan University of Medical Sciences. Iran J Ergon. 2020;8(2):17-25. [DOI:10.30699/jergon.8.2.17]

15. Pierce JL, O'driscoll MP, Coghlan AM. Work environment structure and psychological ownership: The mediating effects of control. J Soci Psychol. 2004;144(5):507-34. [DOI:10.3200/SOCP.144.5.507534] [PMID]

16. Cameron KS, Quinn RE. Diagnosing and changing qrganizational culture. Based on the competing values framework. Reading, MA: Addison-Wesley.1999.

17. Downs C, Adrian A. Communication audits. lawrence, KS: Commun Manag. 1997.

18. Bass BM, Avolio BJ. Manual: The multifactor leadership questionner. J Consulting Psychologists Press, Palo Alto, CA. 1990.

19. Porter LW, Steers RM, Mowday R. Organizational commitnent. J Appl Psychol. 1983;59(5):603-9. [DOI:10.1037/h0037335]

20. Spretzer GM. Psychological empowerment in the work place: Dimensions. Meas Valid, Acad Manag J. 1997:38:1442-65. [DOI:10.5465/256865]

21. Avey JB, Avolio BJ, Crossley CD, Luthans F. Psychological ownership: Theoretical extensions, measurement and relation to work outcomes. J Organizational Behavior: Int J Industri Occup Organ
Psychol Behav. 2009; 30(2): 173-91. [DOI:10.1002/job.583]

22. Paterson DG. The scott company graphic rating scale. J Pers Res. 1963; 1:361-376.

23. Hosseini ST. Investigating the organizational factors affecting the empowerment of human resources in the Deputy Minister of Development of the Ministry of Jihad Agriculture. J Educ Manag Res 2013;5(1):35-54.

24. Malakouti E, Yazdani B. The role of charismatic leadership on organizational commitment: The Moderating Impact of Value Reduction (Case Study: Isfahan Mobile Telecommunications Company). International Conference on Innovation in Business and Econ Manag, Tehran, Iran Bus Excell Assoc. 2018.

25. Jaffery $H$, Farooq $H$. The impact of structural empowerment on organizational citizenship behaviororganization and job performance: A mediating role of burnout. Burn J Manag. 2015;2(2):274-89. [DOI:10.20547/jms.2014.1502204]

26. Pingo JC, Dixon MR, Paliliunas D. Intervention enhancing effects of acceptance and commitment training on performance feedback for direct support professional work performance, Stress, and job satisfaction. Behav Anal Pract. 2020;13(1):1-10. [DOI:10.1007/s40617-019 00333-w] [PMID] [PMCID]

27. Mathew GC, Prashar S, Ramanathan HN, Pandey UK, Parsad C. Impact of religiosity, spirituality, job satisfaction and commitment on employee performance: a quantile regression approach. Int $\mathrm{J}$ Indian Cult Bus Manag. [DOI:10.1504/IJICBM.2019.104797]

28. Tadampali AC, Hadi A. The effect of job satisfaction and organizational commitment on work engagement and performance. Adv Soci Sci, Educ Human Res. 2017;149:55-7. [DOI:10.2991/icest-17.2017.19]

29. Huili Ye. Relationship between Employee Personality and Job Performance: Based on the mediating effect of organizational trust and work values .Proceedings of the 2nd international conference on economics and management, education, humanities and aocial sciences. Book Series: Adv Soci Sci Educ Human Res. 2018; 151:110-6.

30. Liu F, Chow IH.S, Xiao D, Huang M. Cross-level effects of HRM bundle on employee well-being and job performance: The mediating role of psychological ownership. Chin Manag Stud. 2017;11(3):520-37. [DOI:10.1108/CMS-03-2017-0065]

31. Farzad L, Ranjbar M. Investigating the Relationship between Transformational Leadership and Employee Performance through the Mediating Variable of Psychological Ownership: A Case Study of School Principals, Employees of Aq Qala Education Headquarters. International Conference on New Approaches to the Humanities in the 21st Century. 2016; Rasht, Conference Secretariat. 